\section{P.3.30 UNDERSTANDING THE IMPACT OF PSYCHIATRIC MORBIDITY ON EMPLOYMENT AND BENEFIT CHANGES: A DATA LINKAGE PROJECT}

Sharon Stevelink*, Nicola Fear, Matthew Hotopf. King's College London, London, UK

\subsection{6/OEM-2019-EPI.285}

In the UK the largest cause of long term occupational disability is mental disorders. Closing the disability employment gap has become a focus for recent UK government social policy initiatives, as those being disabled are 32\% less likely to be in employment compared to non-disabled people. Long-term sickness absence is associated with social exclusion, poorer health outcomes and increased mortality. Despite the extensive costs to society and the large number of people adversely affected by this issue, surprisingly little is known about the underlying health reasons for the receipt of benefits and the effectiveness of interventions to support people's return to work. We will describe a novel study that will accelerate our understanding about the dynamics between welfare, benefits and occupational needs of patients presenting with a mental health disorder. This will be done by linking and analysing the Department for Work and Pension data with the South London Maudsley mental health electronic data resulting in the largest clinical cohort of adults $(n=380,000)$ referred to psychiatric services in the UK. The challenges and opportunities accompanying a data linkage project on such a substantial scale will be explored, a description of the linked data will be given and an overview of work currently being conducted.

\section{P.3.33 KNOWLEDGE, ATTITUDES, BEHAVIOR TOWARD NEEDLE STICK INJURIES AMONGST JUNIOR DOCTORS IN A SINGAPOREAN HOSPITAL - A MIXED-METHODS APPROACH}

${ }^{1}$ Ming Wu Warren Ong*, ${ }^{2}$ See Ming Lim, 1,2Judy Sng, ${ }^{1}$ Jeff Hwang. ' ${ }^{1}$ Saw Swee Hock School of Public Health, National University of Singapore, Singapore, Singapore; ${ }^{2}$ Occupational Health Clinic, National University Health Systems, Singapore, Singapore
Introduction Needlestick injuries (NSI) are common healthcarerelated injuries, and possible repercussions include blood-borne infections. Despite that, a large proportion of NSIs are not reported. This study aims to estimate the prevalence of underreporting, and to evaluate the knowledge, attitude and behavior towards NSIs amongst junior doctors in a Singaporean tertiary hospital.

Methods An explanatory sequential mixed-methods design was employed. Quantitative data were collected through questionnaires completed by 100 junior doctors (response rate 66.7\%). 99 survey responses (1 had missing data) were tabulated. Descriptive statistics and bivariate analysis were performed to analyse socio-demographic characteristics, NSI history, and NSI reporting practices. Qualitative data were collected through 12 in-depth interviews. Participants were purposively recruited and semi-structured topic guides were developed using the Health Belief Model (HBM). Data analysis was done based on a thematic, inductive approach.

Results $51.5 \%$ of respondents had history of NSI. Amongst respondents with past NSI, 31.4\% did not report. NSI reporters were 1.52 times as likely to be aware of how to report as compared to non-reporters $(p=0.033)$. NSI reporters were 1.63 times as likely to feel that reporting benefits their health as compared to non-reporters $(p=0.006)$. NSI reporters were $83 \%$ more likely to report a clean needle injury $(p=0.021)$. For non-reporters, the main reasons for not reporting were low risk of transmission (41.2\%) and lack of time to report (35.2\%). Themes identified in qualitative study include perceived benefits, perceived barriers, perceived threat, cues to action, organisational culture and recommendations. Recommendations suggested were to change organisational culture, change reporting process, provide reminders and improve lessons on reporting.

Conclusion Under-reporting of NSI may have significant implications to patients, healthcare workers themselves and to the system. Solving this will require recognition that under-reporting is a significant public health problem, then subsequently addressing factors, and instituting targeted interventions to improve reporting rate. 